\title{
Guideline for optimization of surgical and pathological quality performance for radical prostatectomy in prostate cancer management: evidentiary base
}

\author{
Joseph L. Chin, MD, FRCSC,; John Srigley, MD, FRCPC, ${ }_{r}^{+}$Linda A. Mayhew, MA, ${ }_{r}^{*}$ R. Bryan Rumble, MSc, \\ CCRA, ${ }^{\S}$ Claire Crossley, CHE, BA, MEd, ${ }^{ \pm}$Amber Hunter, MBA; ${ }^{* *}$ Neil Fleshner, MD, MPH, FRCSC, ${ }_{i}^{+t}$

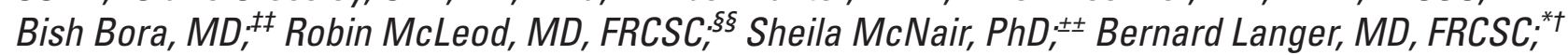 \\ Andrew Evans, MD, PhD, FRCPC; ${ }^{+\neq}$and the Expert Panel on Prostate Cancer Surgery and Pathology
}

See related article on page 26

\begin{abstract}
Background: The objective is to provide surgical and pathological guidelines for radical prostatectomy (RP) with or without concurrent pelvic lymph node dissection (PLND) to achieve optimal benefit for patients, with minimal risk of harm.

Methods: For surgical questions, a literature search of MEDLINE, EMBASE and the Cochrane database was performed. A literature search for the pathological questions was not conducted since the protocol for invasive carcinomas of the prostate gland developed by the College of American Pathologists (CAP) was endorsed. Urologists and pathologists were consulted for their assessment of the surgical and pathological recommendations.

Results: Limited high-quality evidence from 95 primary studies was available and, therefore, the expert panel developed recommendations on the basis of a consensus of the expert opinion of the working group and through a consultation with urologists and pathologists. In addition to the CAP protocol, some technical recommendations related to the handling and processing of the specimen were made.

Conclusion: Radical prostatectomy is recommended for the surgical treatment of prostate cancer, depending on a patient's preoperative risk profile. The panel unanimously determined that the goals for RP are to attain a positive margin rate of $<25 \%$ for $\mathrm{pT} 2$ disease, a mortality rate of $<1 \%$, rates of rectal injury of $<1 \%$ and blood transfusion rates of $<10 \%$ in non-anemic patients. Standard PLND should be mandatory in high-risk patients, should be recommended for intermediate-risk patients and should be optional for low-risk patients. The quality and effectiveness of this treatment and of subsequent patient care depend on good management, effective communication and reporting between surgeons and pathologists working together as part of a multidisciplinary team. The complete guideline document is posted on the Cancer Care Ontario website (www.cancercare.on.ca); search in their Toolbox, Quality Guidelines \& Standards, Clinical Program category under "surgery."
\end{abstract}

Can Urol Assoc J 2010;4(1):13-25

\section{Résumé}

Contexte : Notre objectif était de fournir des lignes directrices sur les plans chirurgical et pathologique pour la prostatectomie radicale (PR) et l'excision concomitante de ganglions lymphatiques pelviens, en vue d'optimiser les bienfaits pour les patients et de minimiser les risques de complications.

Méthodologie : Sur le plan chirurgical, une recherche d'article a été effectuée dans les bases de données MEDLINE, EMBASE et Cochrane. Aucune recherche d'article n'a été effectuée sur le plan pathologique puisqu'il a été décidé $\mathrm{d}^{\prime}$ adhérer au protocole sur les carcinomes invasifs de la prostate élaboré par le College of American Pathologists (CAP). On a ensuite demandé à des urologues et des pathologistes d'évaluer les recommandations.

Résultats : Les résultats de 95 études primaires ont fourni des données de haute qualité limitées et par conséquent, le groupe d'expert a élaboré ses recommandations à partir d'un consensus sur la base des opinions du groupe de travail et à la suite de consultations avec des urologues et des pathologistes. En plus du protocole du CAP, des recommandations techniques liées à la manipulation et au traitement des échantillons ont été formulées.

Conclusion : La PR est recommandée pour le traitement chirurgical du cancer de la prostate en fonction du profil de risque préopératoire du patient. Le groupe d'experts a déterminé d'un commun accord que les objectifs de la PR sont les suivants : taux de marges positives $<25 \%$ dans les cas de tumeurs pT2, taux de mortalité $<1 \%$, taux de complications rectales $<1 \%$ et taux de transfusions sanguines $<10 \%$ chez les patients non anémiques. Une excision standard des ganglions lymphatiques pelviens devrait être obligatoire chez les patients à risque élevé, recommandée chez les patients à risque intermédiaire et facultative chez les patients à faible risque. La qualité et l'efficacité de ce traitement et des soins subséquents dépendent d'une bonne prise en charge et d'une communication efficace entre chirurgiens et pathologistes au sein d'une équipe multidisciplinaire. Les lignes directrices complètes se trouvent sur le site Web d'Action Cancer Ontario (www.cancercare.on.ca); en anglais seulement: dans le Toolbox, Quality Guidelines \& Standards, Clinical Program category "surgery." 
Chin et al.

T he number of newly diagnosed cases of prostate cancer in Canada is increasing as a result of an aging population, increased public awareness, and the widespread use of prostate-specific antigen (PSA) as a tool for prostate cancer screening and early detection. ${ }^{1,2}$ Recent projections from Cancer Care Ontario (CCO) administrative data show that the incidence of prostate cancer in Ontario will increase from 9900 cases in 2005 to almost 13500 cases in 2010. The proportion of early-staged cancers has also increased because of these factors. While radical prostatectomy (RP) is only one of the several management options for localized disease in Ontario, about 3000 RPs are performed per year, and this number is expected to increase with the demand for early-stage treatment. The main goals of RP are (a) complete eradication of the cancer-containing organ with negative surgical margins, thereby maximizing the chance of cure, (b) preservation of urinary function, and (c) preservation of erectile function, where appropriate, but, in some cases, it is not possible to achieve all these goals.

The effectiveness of RP in the treatment of prostate cancer depends on good surgical and pathological management and on the effectiveness of communication between the surgical and pathological teams and other cancer care providers. Proper handling of the specimen in the operating room and complete and clear communication of information in the accompanying requisition form provide the starting point for high-quality pathological analysis and reporting of results to the surgeon and other care providers. The pathological assessment of prognostic factors (e.g., Gleason score, pathologic stage, margin status) is best accomplished through systematic handling of the surgical specimen. ${ }^{3}$ Clear and unambiguous communication of the results (particularly the prognostic factors) in the pathology report are essential for planning the subsequent treatment and care of the individual patient, for assessing the quality of surgical management (margin status), and for system planning purposes. Therefore, to attain the highest quality treatment and management for prostate cancer, both surgical and pathological procedures need to be well-integrated.

Most RPs in Ontario are currently performed by the open retropubic route; however, robotic-assisted and laparoscopic prostatectomy (LP) are being increasingly performed in some centres. Radical prostatectomy is a technically challenging oncologic procedure that requires adequate prior training and proper patient selection. The expectations and outcomes for surgery are the same, regardless of the approach. Pelvic lymph node dissection (PLND) has been commonly used to determine stage in the TNM system. Current practice in Ontario includes PLND for some but not all patients undergoing RP.

The objective of this document is to provide guidelines for surgical techniques for RP and concurrent PLND and for the handling of the surgical specimens in the operating room and laboratory in order to achieve optimal benefit for the patient with minimal risk of harm. This document does not deal with the choice of management options for early-staged prostate cancer. The assumption is that a detailed discussion with the patient regarding treatment options and various techniques for performing prostatectomy, appropriate to the given disease grade and stage, has already taken place. Neither salvage prostatectomy (following local radiotherapy failure) nor the role of neoadjuvant hormonal therapy in RP is addressed in this document.

\section{Questions}

\section{Surgical questions}

What are the recommended surgical procedures and outcomes for RP, specifically:

1. What is the recommended extent of resection, and what is an acceptable positive margin rate?

2. What are the reported rates for surgical complications, specifically incontinence, erectile dysfunction, rectal injury, and blood transfusion, and does surgical technique (e.g., nerve sparing, bladder neck preservation) affect complication rates?

3. Under what circumstances should nerve-sparing techniques be used?

4. Which patients should receive PLND, and what is the recommended extent of PLND?

\section{Pathological questions}

1. What are the recommended procedures for handling the RP specimen in the operating room and for handling and processing the RP specimen (with or without lymph nodes) in the pathology laboratory?

2. What diagnostic and prognostic elements should be included in the pathology report, what format should be used, and what reporting elements should be included?

\section{Definitions used in this document}

\section{Positive surgical margin}

The microscopic presence of a tumour at the inked margin of the surgically excised specimen. ${ }^{4}$

\section{Clinically localized disease}

Defined by digital rectal examination findings and/or bone scan and abdominal and pelvic computerized tomography $(\mathrm{CT})$, as confined to the prostate, and no clinical evidence of extraprostatic disease. ${ }^{5,6}$ 


\section{Risk categories}

Patients may be considered "low", "intermediate" or "high" risk for treatment failure (e.g., local recurrence, biochemical failure with PSA relapse, emergence of metastatic disease) based on disease characteristics, using the definitions proposed by D'Amico and colleagues: ${ }^{7}$

- Low risk: PSA $<10$, Gleason $\leq 6$ and clinical stage T1 or T2

- Intermediate risk: PSA 10-20 and/or Gleason 7

- High risk: PSA $>20$, Gleason $\geq 8$ or clinical stage $\geq T 3$

\section{Methods}

This guideline, posted on the CCO website, was developed by $\mathrm{CCO}^{\prime}$ s Program in Evidence-Based Care (PEBC) using the methods of the Practice Guidelines Development Cycle. ${ }^{8}$ This report, produced by CCO's Surgical Oncology Program (SOP) and the PEBC, is a convenient and up-todate source of the best available evidence on surgical and pathological standards for prostate cancer surgery, developed through a systematic review of the available evidence. The PEBC is supported by the Ontario Ministry of Health and Long-Term Care (MOHLTC) through CCO. The SOP and the PEBC are both editorially independent of CCO and MOHLTC.

Cancer Care Ontario and the Expert Panel on Prostate Cancer Surgery and Pathology endorse the protocol for invasive carcinomas of the prostate gland developed by the College of American Pathologists (CAP). ${ }^{3,4}$ Since the questions of interest for this guideline are addressed in the CAP protocol, a literature search was not conducted for the pathological questions.

\section{Literature search strategy}

MEDLINE and EMBASE databases were searched for evidence related to the surgical questions in March 2007, using the following text, MeSH, and EMBASE subject headings: "prostatic neoplasms," "prostate cancer," "prostate tumour" and other relevant key terms. For more information on these key terms, consult the Evidence-based Series document on prostate cancer management on the CCO website. ${ }^{9}$

The total results were limited to human studies in the English language published from 1996 through to March 2007. These searches produced 5311 references. Similar methods were employed to search for evidence-based reviews and clinical practice guidelines. A separate search of the Cochrane database was also conducted, using the term "prostatectomy." Inclusion and exclusion criteria are listed in the $\mathrm{CCO}$ document. $^{9}$

\section{Synthesizing the evidence}

Due to the anticipated noncomparative sources of evidence in this report, no pooling was planned.

\section{Consultation with urologists and pathologists}

Formal consensus methods were not employed in the development of this guideline. Ontario urologists and pathologists were consulted in October 2007, prior to the completion of the draft document, to obtain feedback on the recommendations drafted by the working group. The consultation included an email survey and an in-person meeting to discuss the draft recommendations along with current data regarding RP performance in Ontario. The number of participants, survey results and the opinions expressed at the in-person meeting are detailed in the $\mathrm{CCO}$ document, ${ }^{9}$

\begin{tabular}{|c|c|c|c|c|c|}
\hline Topic & $\begin{array}{c}\text { No. of } \\
\text { MEDLINE hits }\end{array}$ & $\begin{array}{c}\text { No. of } \\
\text { EMBASE hits }\end{array}$ & $\begin{array}{l}\text { No. ordered for full- } \\
\text { publication review }\end{array}$ & $\begin{array}{l}\text { No. of articles } \\
\text { included in this report }\end{array}$ & $\begin{array}{l}\text { Table } \\
\text { number }\end{array}$ \\
\hline \multicolumn{6}{|l|}{ Radical prostatectomy } \\
\hline Margins & 189 & 479 & 56 & 39 & 4 \\
\hline Complications & 1997 & 2285 & 31 & 22 & 5 \\
\hline $\begin{array}{l}\text { Guidelines/ } \\
\text { systematic reviews }\end{array}$ & 7 & 0 & 0 & 0 & - \\
\hline Cochrane reviews & 13 & - & 0 & 0 & - \\
\hline \multicolumn{6}{|l|}{ PLND } \\
\hline PLND & 327 & 34 & 101 & 23 & 6 \\
\hline
\end{tabular}


Chin et al.

and summarized in the results section following the review of the evidence from the literature for each question.

\section{Results: surgical questions}

\section{Literature search results}

The results obtained from the systematic literature review are shown in Table 1.

\section{Systematic reviews and guidelines}

A total of 20 potentially relevant clinical practice guidelines and evidence-based reviews were found and are described in the CCO document. ${ }^{9}$ None of the 7 guidelines or systematic reviews identified in the MEDLINE or EMBASE literature search was considered relevant. Thirteen Cochrane reviews were found, but all were considered to be outside the scope of this document.

\section{Primary studies}

For the surgical questions, an initial sort of the 5311 citation and abstract results was performed by one of the authors (LM), using the inclusion and exclusion criteria specified in the methods section. The remaining 904 references were then reviewed by another author (JC), and 188 potentially relevant studies were ordered for full-publication review. These 188 studies were reviewed for relevancy by 2 other authors (SM and LM), and 95 were retained for inclusion in this report. Reasons for exclusion are listed in the CCO document. $^{9}$

\section{Study quality}

No randomized controlled trials (RCTs) were located that were designed to specifically determine how the extent of resection and lymphadenectomy, resection margins, continence outcomes or management of neurovascular bundles are related to survival or other outcomes, and owing to ethical considerations, it is unlikely that such studies will become available in the future. One RCT compared limited to extended PLND. For this reason, most of the evidence reviewed for these recommendations is based on retrospective reviews, databases, case series and non-randomized prospective studies, often without comparison groups. These study designs are inherently more biased than randomized studies, and may be difficult to interpret and compare. Confounding factors, such as neoadjuvant or adjuvant therapy and patient baseline characteristics, were not always reported and the surgical techniques used often varied from study to study. The subsequent parts of this guideline high- lights the best available evidence, with respect to the questions posed. The evidence provided context and some direction for the development of recommendations, based on the expert opinion of the panel.

\section{Surgical questions}

\section{Question 1: What is the recommended extent for resection, and what is an acceptable positive margin rate?}

The goal of resection is a negative surgical margin (-SM), thereby hopefully increasing the likelihood of a cure. A summary of the studies is listed in Table 3 in the CCO document. ${ }^{9}$ Seven studies with sample sizes of $\geq 1000$ reported higher recurrence rates for positive margins versus negative margins and/or multivariate analyses showing margin status to be a significant predictor of biochemical recurrence. No data are available for the impact of positive surgical margin status on metastasis-free, disease-specific or overall survival.

In total, 39 case-series studies (36 retrospective and 3 prospective) reporting on positive surgical margins (+SM) were included in the evidence review for this question. Bias is inherent in case series but may be somewhat minimized by a larger sample size. The studies are summarized in Table 4 in the CCO document. ${ }^{9}$

\section{Overall +SM rates}

Overall +SM rates varied from $4.0 \%{ }^{10,11}$ to $45.2 \%{ }^{11}$ for open surgery. The only laparoscopic study that reported an overall + SM reported a rate of $16.7 \% .{ }^{12}$

\section{Clinical stage, Gleason score and $+S M$}

Three studies reported + SM rate by clinical stage. ${ }^{13-15}$ The +SM rates reported were $0 \%{ }^{15}$ to $37 \%{ }^{14}$ for $\mathrm{CT} 1$ and $9.2 \%{ }^{15}$ to $44 \%^{14}$ for cT2 disease. Only one study reported a rate for cT3 $(22.4 \%) .{ }^{15}$ Nine studies reported +SM rate by Gleason score. ${ }^{13-21}$ In general, +SM rates for Gleason 2-6 ranged from $4.2 \%{ }^{18}$ to $31 \%,{ }^{20}$ Gleason 7 ranged from $9.8 \%{ }^{18}$ to $41 \%,{ }^{20}$ and Gleason $8-10$ ranged from $17.7 \%{ }^{18}$ to $71.4 \%{ }^{21}$ Details are listed in Table 4 in the CCO website. $^{9}$

\section{Pathological stage and $+S M$}

Rates for $+\mathrm{SM}$ by pathological cancer stage were compared in 12 studies..$^{12-15,19,21-27}$ In general, the +SM increased with the pathological stage, with ranges from $0 \%{ }^{23}$ to $24 \%{ }^{14}$ for pT2 $\left(3.3 \%^{12}\right.$ to $19.2 \%{ }^{24}$ for those receiving laparoscopic surgery), $24.2 \%^{25}$ to $64.3 \%^{14}$ for pT3a $\left(30 \%{ }^{13}\right.$ to $33 \%{ }^{12}$ for laparoscopic), $27.1 \%^{25}$ to $80.0 \%^{14}$ for pT3b $\left(32 \%{ }^{13}\right.$ to 
$47 \%{ }^{12}$ for laparoscopic), and $16.7 \%^{23}$ to $40.0 \%{ }^{14}$ for pT3c. Three further studies ${ }^{16,20,28}$ reported + SM by $\mathrm{T}$ stage, but as it was unclear as to whether these were clinical or pathological stage, these data are not included here.

\section{Margin site and +SM rates}

Ten studies reported the location of positive margins. ${ }^{16,19,21,22,27,29-33}$ Reported apical + SM rates ranged from $8 \%{ }^{30}$ to $58 \%,{ }^{29}$ posterior $+\mathrm{SM}$ ranged from $9 \%{ }^{22}$ to $40 \%,{ }^{29}$ anterior + SM ranged from $1.2 \%{ }^{31}$ to $15 \%,{ }^{16}$ base + SM ranged from $2 \%{ }^{19}$ to $19 \%,{ }^{29}$ and bladder neck + SM rates ranged from $4 \%{ }^{30}$ to $20.9 \% .^{27}$ Five studies reported the location of the positive margin by the stage of disease. ${ }^{14,26,27,34,35}$ One study of laparoscopic RP reported that $50 \%$ of + SM were apical, $30 \%$ were posterolateral, and $20 \%$ occurred at the prostate base. ${ }^{13}$ A second laparoscopic study found $40.3 \%$ of + SM were posterolateral, $26.1 \%$ were apical, $6.2 \%$ were anterior and $6.2 \%$ were at the bladder neck. ${ }^{24}$

\section{Surgical technique and $+S M$}

Eight studies compared +SM rates for nerve-sparing surgery versus non-nerve sparing or nerve-sparing versus wide excision. ${ }^{14,24,26-28,30,32,33}$ This topic is discussed further in the section below under Question 3 related to nerve sparing surgery.

\section{Surgeon and +SM}

While we did not include many studies that specifically addressed differences in +SM by surgeon, Eastham and colleagues noted that the + SM rate ranged from $10 \%$ to $48 \%$, depending on the surgeon. ${ }^{17}$

\section{Consultation with urologists and pathologists}

\section{Survey questions and responses}

- The positive resection margin for pT2 ranges from $0 \%$ to $53 \%$ across Ontario. In your opinion, is this acceptable? Yes $=5(11.6 \%)$, No $=38(88.4 \%)$

- The incidence of positive surgical margins should be $<20 \%$ for pT2 disease. Agree = $33(75 \%)$, No $=5(11.4 \%)$

- In high-risk patients, a positive surgical margin rate in the range of $35 \%$ should be achievable. Agree $=43$ $(55.8 \%)$, Disagree $=12(27.9 \%)$

\section{Discussion}

Most participants agreed that the current provincial average should be improved and that an average of $25 \%$ is a reasonable target for pT2 patients. The issues raised included the fact that defining a benchmark rate is difficult because many factors affect $+\mathrm{SM}$ rates.

Question 2: What are the reported rates for surgical complications, specifically incontinence, erectile dysfunction, rectal injury, and blood transfusion; and does surgical technique (e.g., nerve sparing, bladder neck preservation) affect complication rates?

A total of 22 studies were located (Table 5 in the CCO document ${ }^{9}$ ), including 1 randomized trial that compared rectal injury rates and blood transfusion rates for radical retropubic prostatectomy (RRP) to rates for LP. ${ }^{36}$ Seventeen studies were retrospective case series, 3 were prospective case series, and 2 were cross-sectional surveys administered after surgical interventions. Bias is inherent in these study designs but may be somewhat minimized by a larger sample size. Study size ranged from 100 to 10737 , and 10 studies had sample sizes of more than 500 subjects.

Perioperative mortality rates reported in 8 studies ranged from $0 \%$ to $0.5 \%$. Overall rates of postoperative complications were reported in 5 studies, ranging from $6.3 \%$ to $28.6 \%$, but the complications included in these rates varied among studies and were unclear in some. The largest study $(\mathrm{n}=$ 10 737) reported statistically significant variation among 159 high-volume surgeons with respect to complication rates. ${ }^{37}$ Another study of 3477 patients undergoing RP with 1 surgeon from 1983 to 2003 found that complications rates dropped over time from $16.9 \%$ (1983-1991) to $7.4 \%$ $(1992-2003){ }^{38}$

\section{Urinary function}

Sixteen studies reported on incontinence. The results of these studies are difficult to interpret because incontinence was defined and assessed using different criteria, ranging from "any degree of loss" to the use of 4 or more pads daily. Some reported rates were related to the time post-surgery of 12 or 24 months and some to the age of the patients, while some reported daytime versus nighttime incontinence or combinations of these. In general, the reported incontinence rates ranged from $5 \%{ }^{39}$ to $67 \%,{ }^{40}$ and those for more severe incontinence ranged from $0.8 \%$ to $20 \%$. One study reported a decline in incontinence rates from 12 to 24 months post-surgery, ${ }^{39}$ and another reported a higher rate for men aged over $70 .{ }^{41}$

Four studies compared continence rates for various surgical techniques. Incontinence rates were $1.3 \%$ with bilateral nerve-sparing surgery (BNS), 3.4\% with unilateral nervesparing surgery (UNS) and $13.7 \%$ with non-nerve-sparing surgery. ${ }^{42}$ Bladder neck preservation reduced incontinence rates at 12 months to $10.6 \%$ from $13.7 \%$ for bladder neck resection, ${ }^{43}$ and when both bladder neck-sparing and 
Chin et al.

puboprostatic ligament-sparing techniques were employed, the incontinence rate at 12 months was $6 \%$ compared to $8 \%$ for either technique alone. ${ }^{30}$ Incontinence rates at 12 months were lower for laparoscopic surgery compared to open RRP, with rates of $11.0 \%$ versus $22.3 \%$ for diurnal incontinence and $4.0 \%$ versus $10.0 \%$ for nocturnal incontinence. ${ }^{44}$

\section{Erectile function}

This topic is covered in the section on neurovascular bundles.

\section{Rectal injury}

Seven studies reported rates of rectal injury ranging from $0.3 \%$ to $1.45 \%$ for RRP and $1.7 \%$ for LP. ${ }^{12,36,41,45-48}$ One study found higher rates when a perineal approach was used, compared to a retropubic approach $(p=0.03) .{ }^{46}$

\section{Blood transfusion}

Seven studies ${ }^{12,36,46-50}$ reported blood transfusion rates ranging from $1.4 \%{ }^{46}$ to $67 \% .^{48}$ One study reported a median value of 3 units of blood used; ${ }^{47}$ another reported an average of 2.13 with a range of 1 to 7 units. ${ }^{49}$ Rates were lower for LP than for RRP for both homologous ( $0 \%$ vs. $9 \%$ ) and autologous ( $13.3 \%$ vs. $45 \%$ ) transfusion. ${ }^{36}$

\section{Consultation with urologists and pathologists}

\section{Survey questions and responses}

- An acceptable rate for rectal injury should be $<1 \%$. Yes $=42(100 \%), \mathrm{No}=0$

- An acceptable rate for blood transfusion should be $<10 \%$. Yes $=38(88.4 \%)$, No $=4(9.3 \%)$

\section{Discussion}

The blood transfusion rate should apply to non-anemic patients. The operation time frame and indications for transfusion should also be considered.

\section{Question 3: Under what circumstances should nerve-sparing techniques be used?}

Various nerve-sparing techniques have been developed in an attempt to preserve potency in as many patients as possible. In the past, an assumption was made that using nervesparing techniques compromised cancer control, so their use has been controversial. There is also some controversy concerning whether preserving neurovascular bundles may also lead to increased continence rates.

\section{Nerve-sparing surgery and positive margin rate}

Neurovascular bundles are excised more often in men with higher grade disease, ${ }^{16}$ and patients in the nerve-sparing groups are also often younger and have a lower PSA, ${ }^{32}$ making comparisons between the 2 patient groups difficult. Information concerning nerve-sparing surgery and positive margin rates is available in Table 4 in the CCO document. ${ }^{9}$

Graefen and colleagues noted that there was a higher positive margin rate for non-nerve-sparing surgery, particularly in pT3c cancers, but that there were no statistically significant differences in the incidence of biochemical relapse, even when an "ultra-sensitive" PSA test was used. ${ }^{23}$ Palisaar and colleagues also found higher positive margin rates for those who received non-nerve-sparing surgery for pT3 grade cancer, and noted that the 5-year biochemical recurrence-free survival was higher for those who received nerve-sparing surgery. ${ }^{26}$

Rabbani and colleagues reported that there was no significant difference in positive apical margin rates for patients undergoing bilateral, unilateral, or non-nerve-sparing surgery, when the patients were stratified by clinical stage or the presence of perineural invasion. ${ }^{14}$ Cannon and colleagues found that, in 61 patients with nerve-sparing surgery on a single side, only one had a positive surgical margin. ${ }^{51}$ Of the 57 patients who had both nerve bundles spared, only 4 had positive margins, and only one of those margins occurred on the same side as the perineural invasion. Sofer and colleagues found that patients who received nervesparing surgery were not at an increased risk of recurrence compared with non-nerve-sparing patients (hazard ratio [HR] 0.96, 95\% confidence interval [Cl] 0.53-1.72) when adjustments were made for positive surgical margins, PSA, Gleason, seminal vesicle invasion, T stage, capsular involvement, extraprostatic extension, and age. ${ }^{32}$

In a large retrospective study of 7268 men, Ward and colleagues controlled for age, clinical stage, biopsy grade, year of surgery, and PSA levels, and found that nerve-sparing surgery had no significant impact on biochemical progression rates (HR $0.98,95 \% \mathrm{Cl} 0.88$ to $1.08, p=0.64) .{ }^{33}$ The rate of positive surgical margins was actually lower (odds ratio [OR] $0.86,95 \% \mathrm{Cl} 0.76$ to $0.97, p=0.012$ ) in those who received nerve-sparing surgery.

\section{Erectile function}

Ten studies reported on erectile function, and the information concerning erectile function can be found in Table 5 in the CCO document. ${ }^{9}$ The reported potency rates ranged from $48 \%{ }^{52}$ to $91.8 \%{ }^{46}$ of patients. One large study $(\mathrm{n}=$ 5238) reported a median time of 12 months to recover erectile function and an increase of $7 \%$ from 18 months to 24 months. ${ }^{53}$ Three studies found that BNS resulted in higher 
rates of erectile function than did UNS, with differences of $23 \%, 21 \%$, and $7 \%$, respectively. ${ }^{22,38,41}$ Men 59 years and younger benefited more $(41 \%, 49 \%)$ than men over 60 (10\%, $8 \%) .{ }^{41}$ One study of 300 patients reported higher rates of erectile function for LP compared to RRP (41\% vs. 30\%, respectively) whether one neurovascular bundle (46\% vs. $27 \%$ ) or two (53\% vs. $44 \%$ ) were preserved. ${ }^{44}$ Catalona and colleagues also found that the proportion of men with a return of erectile function increased with the number of prior prostatectomies performed by the surgeon $(61 \%$ for less than 500, 68\% for 500 to 1000 , and $70 \%$ for 1000 to 1,500; Armitage chi-square 4.8, $p=0.03$ ) and that there was a significant interaction for age by type of surgery (Wald chi-square 6.9, $p=0.009$ ), with the effect of BNS versus UNS on the odds of regaining potency decreasing with increasing age. ${ }^{41}$

\section{Continence}

The role of nerve-sparing surgery in the recovery of continence is controversial. Detailed information concerning continence and nerve-sparing surgery can be found in Table 5 in the CCO document. ${ }^{9}$ Graefen and colleagues, ${ }^{23}$ Kundu and colleagues, ${ }^{38}$ and Catalona and colleagues ${ }^{41}$ reported that the recovery of urinary incontinence was not associated with nerve-sparing surgery. Burkhard and colleagues, however, found that when age, PSA, pT stage, Gleason, and node-positive status were examined along with type of surgery, attempted nerve-sparing surgery was the only statistically significant factor influencing urinary incontinence (OR 4.77, 95\%, Cl 2.18 to $10.44, p=0.0001) .{ }^{42}$

\section{Consultation with urologists and pathologists}

\section{Survey questions and responses}

- Sparing of the neurovascular bundles should be considered the "standard approach" unless it is contraindicated. Yes $=33(76.7 \%)$, No $=8(18.6 \%)$

- In situations where there is a high risk of positive margins, based on clinical evidence, or the likelihood of extracapsular tumour extension and risk categorization (e.g., clinical stage $>$ T2, Gleason $>7$, high-volume disease, intraoperative finding of induration of lateral pelvic fascia), wide excision of the neurovascular bundles would be warranted in order to avoid compromise to cancer control. Yes $=39(97.5 \%), N_{0}=0$

- Clips should be used for hemostasis, and the use of electrocautery near the neurovascular bundles should be avoided. Yes $=31(81.6 \%), N o=3(7.9 \%)$

\section{Discussion}

There was general agreement that nerve-sparing techniques are appropriate for low-risk patients but should not be performed in high-risk patients or patients who are not sexually active. The decision to use nerve-sparing techniques should be determined a priori, giving consideration to cancer control, risk, potency, and continence, with the caveat that the intraoperative finding of induration of the lateral pelvic fascia might alter the a priori decision. Contraindications include PSA level, amount of high-risk cancer, extracapsular extension, and pathological stage. There was general agreement that in practice, patient selection is based on anecdote and feel in many cases.

\section{Question 4: Which patients should receive pelvic lymph node dissec- tion (PLND), and what is the recommended extent of PLND?}

A total of 22 studies were located: 21 case series (15 retrospective, and 6 prospective) and one randomized trial $(\mathrm{n}=123)^{54}$ in which patients were prospectively randomized to have extended PLND on one side and limited PLND on the other. The case series studies lack controls and are not randomized; they are therefore more susceptible to bias than more robust study designs such as RCTs However, a case series with a large sample size is more robust than one with a small sample size. In these studies, sample size ranged from 123 to 9182, and 6 studies had sample sizes of more than 1000 subjects. The results of these studies are reported in Table 6 in the full document on the CCO website. ${ }^{9}$

Other factors affect the quality of the evidence found. In retrospective studies, there is no control over patient selection, and so patients who received PLND or extended PLND may have been those considered to be at higher risk. As mentioned by Briganti and colleagues, ${ }^{55}$ many of the patients who underwent extended PLND had higher PSAs and higher Gleason scores, and Berglund and colleagues ${ }^{56}$ noted that the treatment and no-treatment groups were statistically significantly different in age and disease stage. In addition, little information is available as to how patients were picked for extended versus limited PLND, making comparisons between these groups difficult. The staging methods used in these studies are also inconsistent, as some used Gleason scores, some used PSA values, some used clinical TNM, some used pathological TNM, and some used various combinations of these. Further, PSA tests have also become more common and more sensitive over time, which may be leading to a stage migration in the diagnosis of prostate cancer.

\section{Therapeutic value}

In some other cancers, such as testicular non-seminoma, lymphadenectomy has proven beneficial to the patient; 
Chin et al.

however, the therapeutic value of removing pelvic lymph nodes in prostate cancer is not well established. Seven studies in this review addressed the therapeutic role of PLND in treating prostate cancer patients: 3 supported, and 4 rejected, a therapeutic value for PLND. All these studies were retrospective case series.

In one study of 9182 patients who underwent PLND, patients who had more than four lymph nodes examined showed a significant decrease in HR for cancer-specific death, and for patients with negative nodes, the HR for cancerspecific death increased significantly when more than 10 nodes were removed. ${ }^{57}$ Removing a large number of lymph nodes in node-negative men improved neither the HR for death ${ }^{57}$ nor the biochemical recurrence rate. ${ }^{58}$ In another study, patients with nodal involvement and less than $15 \%$ positive nodes who underwent extended PLND had a significantly higher PSA progression-free survival rate at five years than those who did not receive PLND. ${ }^{59}$ Three studies, however, did not find any evidence of a therapeutic value for PLND, as performance or omission of PLND was not an independent predictor of outcome. ${ }^{56,60,61}$ DiMarco and colleagues ${ }^{62}$ also found that the number of nodes excised in PLND was not significantly associated with PSA progression, systemic progression, or cause-specific survival.

\section{Staging}

Of twenty studies identified that addressed the benefit of using PLND for staging, 11 supported, and 8 rejected performing a PLND or an extended PLND, and one study provided information supporting both sides of the issue. Six of these studies were prospective; 5 supported PLND and 1 rejected PLND.

Four studies ${ }^{63-66}$ found that patients would be understaged without a PLND, particularly low-risk patients. ${ }^{65}$ Pagliarulo and colleagues found the presence of occult lymph node metastases in $13.3 \%$ of patients. ${ }^{67}$ Rogers and colleagues ${ }^{68}$ found that other preoperative factors, such as Gleason and PSA, were not sufficiently sensitive to predict who would have nodal metastases, and Bader and colleagues ${ }^{63}$ found that CT imaging has low sensitivity and accuracy for lymph node metastases.

Other studies have not found PLND to be an important part of staging. Three studies ${ }^{55,68,69}$ found that other clinicbiological factors could identify patients with an increased risk of positive lymph nodes. ${ }^{56,69,70}$ Further, Briganti and colleagues stated that the staging benefit of PLND should be juxtaposed with the higher complication rates and longer hospital stay, especially with extended PLND. ${ }^{55}$

\section{Extent}

In the literature reviewed, there was considerable variation in the reported extent of PLND and the definition of the terms used to describe the extent of surgical removal of tissue. In some studies, standard or limited PLND was compared to extended or "meticulous" PLND or to no PLND, but the descriptions of these terms differed among studies.

Eight studies found positive lymph nodes outside the area of a standard PLND and were in support of performing an extended PLND. 45,59,63,64,70-73 In 3 studies we found that an extended PLND was unnecessary. ${ }^{58}$ In the randomized trial by Clark and colleagues, where patients had a limited PLND on one side and an extended PLND on the other side, there was no difference in the number of positive nodes between the limited and the extended PLND. ${ }^{54}$

\section{Complications in PLND}

Balanced against the potential value of PLND as a staging tool or for therapeutic value is the potential for surgical complications. Bhatta-Dhar and colleagues noted that the complication rate for PLND is about $1 \%$ and that there is a greater likelihood of a complication resulting from PLND $(1 \%)$ than of finding positive lymph nodes $(0.7 \%) .{ }^{60}$ Briganti and colleagues found that the complication rate for extended PLND (19.8\%) was significantly higher than the complication rate for the limited PLND $(8.2 \%$, OR 2.7, $p<0.001)$, that the rate of lymphoceles was higher in the extended PLND group, and that extended PLND also resulted in a significantly longer hospital stay. ${ }^{55}$ In the randomized trial by Clark and colleagues, nearly $77 \%$ of complications were on the side of the extended PLND, while there was no difference in the rate of detection of metastases. ${ }^{54}$

\section{Consultation with urologists and pathologists}

\section{Survey questions and responses}

- PLND should be mandatory in high-risk patients. Yes = $41(97.6 \%)$, No $=1(2.4 \%)$

- PLND should be recommended for the intermediate group. Yes $=41(97.6 \%), \mathrm{No}=2(4.8 \%)$

- Standard PLND should include all lymphatic tissue along the external iliac vein from the lymph node of Cloquet distally to the bifurcation of the common iliac vein proximally, and includes all lymphatic tissue in the obturator fossa. Yes $=32(80 \%)$, No $=8(20 \%)$

- Evidence and opinions on the role of extended PLND in high-risk patients are divided.

Yes $=36(90 \%)$, No $=3(7.5 \%)$

- An extended PLND entails removal of lymph nodes medial and lateral to the internal iliac vessels up to and around the bifurcation of the common iliac artery, with the genitofemoral nerve as the lateral limit. Yes $=34(85 \%)$, No $=2(4 \%)$ 


\section{Discussion}

There was general agreement with the recommendations.

\section{Pathological questions}

The Expert Panel on Prostate Cancer Surgery and Pathology endorses the CAP protocol for invasive carcinomas of the prostate gland, and a literature search was not conducted for the pathological questions. The results of the consultation with urologists and pathologists with respect to the pathological questions are presented for each of the recommendations below. (Note: total responses do not sum to $100 \%$ because some respondents did not answer yes or no, but provided a comment.)

Question 1: What are the recommended procedures for handling the $\mathrm{RP}$ specimen in the operating room, and for handling and processing the RP specimen (with or without lymph nodes) in the pathology lab?

\section{Consultation with urologists and pathologists}

\section{Survey questions and responses}

- Frozen section analysis of the radical prostatectomy specimen (RPS) for margin status is not recommended. Yes $=42(93 \%), \mathrm{No}=0$

- For routine handling, the RPS should be fixed in $10 \%$ neutral buffered formalin or other appropriate fixative. The specimen should be put in an appropriately sized container with a minimum formalin/tissue ratio of 10:1 (i.e., 500 cc formalin for a 50 cc prostate). Yes $=42$ (93\%), No $=0$

- The surgical specimen should be accompanied by an appropriate pathology requisition that includes demographic and other identifying information, relevant clinical data (serum PSA, DRE findings [T1c vs. T2], and Gleason score on biopsy), and a history of neoadjuvant therapy (e.g., hormones). Yes $=42$ (91.3\%), No = 4 (8.7\%)

- The prostate gland should be weighed and measured in three dimensions. Yes $=41(93.2 \%), N_{0}=2(4.6 \%)$

- Seminal vesicles should be measured. Yes $=28(62.2 \%)$, No $=13(33.33 \%)$

- Accompanying lymph node specimens should also be measured and a record made of the number and size of grossly identified nodes. Yes $=38(82.6 \%)$, No $=6(13 \%)$

- The outer aspects of the RPS should be carefully inked to identify the surgical margins. A variety of techniques are suitable, including India ink and multi-coloured dyes. Yes $=43(97.7 \%)$, No $=1(2.3 \%)$
- After appropriate fixation and inking, the distal apical segment should be transected and then serially sectioned, perpendicular to the inked surface. An en face (shave) technique is not recommended at the apex. Yes $=37$ $(86.1 \%), N_{O}=0$

\section{Discussion}

There was general agreement with the recommendations.

\section{Question 2: What diagnostic and prognostic elements should be included in the pathology report, what format should be used, and what reporting elements should be included?}

All the respondents agreed that the following items from the CAP RPS checklist should be included in the pathology report: histological tumour type, Gleason grading, presence/absence of seminal vesicle invasion, presence of extraprostatic extension, $\mathrm{pT}$ and $\mathrm{pN}$ designation and margin status.

Other desirable, although not mandatory, elements:

- Presence of tertiary Gleason patterns. Agree = 86.7\%

- Tumour quantification. Agree = 93.3\%

- Extent of extraprostatic extension. Agree $=91.1 \%$

- Presence/absence of lymphatic (small vessel) invasion. Agree $=84.4 \%$

- Presence/absence of venous (large vessel) invasion. Agree $=82.2 \%$

\section{Discussion}

The main goals of RP include the (a) complete eradication of the cancer-containing organ with negative surgical margins, (b) preservation of urinary function and (c) preservation of erectile function where appropriate. The impact of a positive surgical margin is significant since it is an independent prognostic factor for disease recurrence and an indicator for consideration of secondary therapy. Margins are more likely to be reported as positive in more advanced disease but may also be positive because of variation in surgical or pathologic technique. The rate of positive surgical margins for RP has declined over the last 10 years, from upwards of $50 \%$ in the past to a low of $4 \%$ in some contemporary series. This may be partially owing to "stage migration," with more cases of organ-confined cancer being treated with surgery, and to improved surgical techniques. The incidence of positive surgical margins also varies considerably among individual surgeons and individual institutions, with an association between higher volumes and lower rates of margin positivity. In Ontario, the CCO 2005 data indicated that, among the various LHINs, positive resection margin rates ranged from $16 \%$ to $42 \%$ for pT2 disease and $42 \%$ to $83 \%$ for pT3 disease. In the $2005 / 2006$ CCO 
Chin et al.

Pathology Audit, the average positive margin rates were $32 \%$ for pT2 Gleason $\leq 7$ and $59.0 \%$ for pT2 Gleason $>8$ or pT3. The incidence of postoperative incontinence and erectile dysfunction is more difficult to document, but, as in the case of margins, both tumour stage and surgical technique may play an important role.

\section{Surgical management}

The currently available evidence from the literature on surgical quality performance for RP was limited to case series reports and retrospective reviews without randomization or control groups. In general, the evidence from the published literature alone does not provide a strong basis for recommendations, and, therefore, the expert panel developed recommendations and guidance on technical considerations on the basis of a consensus of the expert opinion of the working group and through a consultation with a group of 44 urologists and pathologists in October 2007.

The goals for good surgical management are negative surgical margins, no adverse effects or complications resulting from surgery, and maintenance of continence and erectile function. The decision to offer surgery to high-risk patients should be made with careful consideration. High-risk patients should be offered a referral for radiation consultation or review at a Multidisciplinary Cancer Conference (MCC).

\section{Surgical margins and extent of radical prostatectomy}

There is a demonstrated association between positive surgical margins and higher rates of biochemical failure and clinical recurrence. The rate of positive surgical resection margins is dependent on the tumour risk category (e.g., preoperative PSA level, biopsy Gleason score, clinical T staging, the number of positive biopsy cores, the percentage of involvement of the biopsy cores), extent of surgical dissection and surgical technique, and also the pathologist's handling and reporting with respect to the surgical specimen. It was the consensus of the expert panel that attaining a positive margin rate of $<25 \%$ for $\mathrm{pT} 2$ disease, without compromising disease control, is an achievable goal. Many factors influence the suitability of patients in the high-risk group for RP, and important factors, such as the tumour risk category, should be considered in the context of an MCC. Higher +SM rates are expected for high-risk patients. Positive margins occur at a higher rate at the prostatic apex than at the posterior, base, or anterior of the prostate, and positive margin rates are lower in early-stage cancer than in late-stage cancer.

\section{Surgical complications}

The reported rates of perioperative mortality in RP are consistently $<0.5 \%$. Incontinence and loss of erectile function are potential negative outcomes of RP that have a serious impact on the long-term quality of life for patients, although initial post-surgery rates appear to decline over time from 12 to 24 months. There is limited evidence that nervesparing surgery, bladder neck preservation and laparoscopic surgery result in lower incontinence rates, but the evidence is difficult to interpret due to the variation in assessment and reporting of continence outcomes. There is some evidence that BNS results in higher rates of erectile function than does UNS and that the benefit was more pronounced in younger men. Based on a consensus of expert opinion, the recommendations of the panel are that:

- Radical prostatectomy should be offered to low-risk and intermediate-risk patients for whom surgery is considered a viable, and possibly the preferred, option.

- The decision to offer surgery to high-risk patients should be made with careful consideration. High-risk patients should be offered a referral for radiation consultation or review at a MCC. The intent of the MCC is to ensure that all suitable treatment options and the most appropriate treatment recommendations are generated for each patient and discussed prospectively with a multidisciplinary team with the knowledge and tools to provide a full array of surgical, systemic and radiation treatments, and supportive and palliative care where applicable. The incidence of positive margins in this patient group is expected to be higher than that for pT2 disease.

- Sparing of the neurovascular bundles should be considered the "standard approach" except for high-risk patients.

- In situations where there is a high risk of positive margins based on clinical evidence, or the likelihood of extracapsular tumour extension and risk categorization (clinical stage $>\mathrm{T} 2$, Gleason $>7$, high-volume disease, intraoperative finding of induration of lateral pelvic fascia), wide excision of the neurovascular bundles would be warranted, in order to avoid compromising cancer control.

- Attaining a positive margin rate of $<25 \%$ for pT2 disease should be an achievable goal.

- Achieving rates of $<1 \%$ for rectal injury and $<10 \%$ for blood transfusion in non-anemic patients are the goals.

\section{PLND}

Pelvic lymph node dissection has been used as a staging tool and possibly, as a treatment for reducing the disease burden in some patients. Available evidence is inconclusive on whether the benefits of performing PLND outweigh the harms. The following recommendations are based on the expert opinion and consensus of the panel, based on the D'Amico low-, intermediate-, and high-risk groups.

- Standard PLND should be mandatory in high-risk patients and is recommended for the intermediate group. PLND is optional for low-risk patients. 
- Evidence and opinions on the role of extended PLND in high-risk patients are divided. The Expert Panel noted that an extended PLND might not always be advisable, owing to the higher potential for surgical complication.

\section{Pathological management}

Clear and effective communication of information among surgeons, pathologists, and other caregivers is necessary in order to achieve optimal results for the patient. The expert panel recommendations are based on the CAP recommendations and protocols for reporting and handling of radical prostatectomy specimens in the operating room and the pathology lab as endorsed by CCO. Some additional technical recommendations related to the handling and processing of the specimen were not addressed in the CAP protocol but were agreed to by the panel (see Table 7.b in the $\mathrm{CCO}$ document ${ }^{9}$ ).

\section{In the operating room}

Frozen section analysis of the radical prostatectomy specimen (RPS) for margin status is not recommended. The handling and sectioning of the fresh specimen may significantly distort tissue and impair the final analysis.

It must be decided whether the RPS is being submitted for research studies/tumour banking or for routine handling.

For research purposes or fresh tumour banking, as there is a rapid degradation of some macromolecules (especially RNA) after devitalisation, the RPS should be immediately transported to the pathology laboratory for appropriate handling as per relevant protocols.

For routine handling, the RPS should be fixed in 10\% neutral buffered formalin or other appropriate fixative in an appropriately sized container with a minimum formalin/ tissue ratio of 10:1.

\section{Other technical considerations}

The panel drafted additional recommendations on surgical and specimen handling in the pathology laboratory of a technical nature, and these are compiled in Table $7 \mathrm{a}$ and $7 \mathrm{~b}$ of the full CCO document. ${ }^{9}$

\section{Conclusion}

The members of the Expert Panel on Prostate Cancer Surgery and Pathology conclude that a patient's risk profile helps to determine the treatment options. The quality and effectiveness of radical prostatectomy and of subsequent patient care depend on good surgical and pathological management and on the effectiveness of the communication and reporting between surgeons and pathologists working together as part of a multidisciplinary team. The primary goal of RP is the complete eradication of the cancer-containing organ, with negative surgical margins, with preservation of urinary function and preservation of erectile function where appropriate.

*Regional Head of Surgical Oncology, London Health Sciences Centre, London, ON; ' Provincial Head, Pathology and Laboratory Medicine, Cancer Care Ontario, Mississauga, ON; 隹esearch Associate, Radiation Medicine Program, DRO, Princess Margaret Hospital, University Health Network, Toronto, ON; §Research Coordinator, McMaster University/Cancer Care Ontario Program in Evidence-based

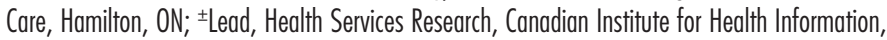
Toronto, ON; ** Program Manager, Surgical Oncology Program, Cancer Care Ontario, Toronto, ON; t†Head of Division of Urology, Princess Margaret Hospital, Toronto, ON; 抽orth East Regional Head

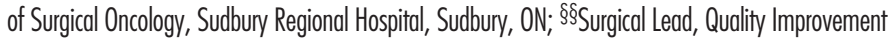
\& Knowledge Transfer, Cancer Care Ontario, Toronto, ON; ${ }^{ \pm}$Assistant Director, McMaster University/Cancer Care Ontario Program in Evidence-based Care, Hamilton, ON; ${ }^{*}{ }^{*}$ Consultant, Cancer Care Ontario, Toronto, ON; † $\$$ Pathologist and Associate Professor, University Health Network, Toronto General Hospital, Toronto, ON

Acknowledgements: Members of the Expert Panel on Prostate Cancer Surgey and Pathology are (in alphabetical order): Dr. Alexander Boag (Pathologist), Kingston General Hospital, Kingston, ON; Dr. Bish Bora (Surgeon), Sudbury Regional Hospital, Sudbury, ON; Dr. Joseph Chin, Chair (Surgeon), London Health Sciences Centre, London, ON; Paul Darby, CEO, Peterborough Regional Health Centre, Peterborough, ON; Dr. Dimitrios Divaris (Pathologist), Grand River Hospital-KitchenerWaterloo Health Centre, Kitchener, ON; Dr. Andrew Evans (Pathologist), University Health Network, Toronto General Hospital, Toronto, ON; Dr. Neil Fleshner (Surgeon), University Health Network, Princess Margaret Hospital, Toronto, ON; Amber Hunter, Program Manager, Surgical Oncology Program, Cancer Care Ontario, Toronto, ON; Dr. Angelo locca (Surgeon), Royal Court Medical Centre, Barrie, ON; Dr. John Kell, President, Society of Urological Surgery in Ontario, Toronto, ON; Dr. Bernard Langer, Consultant, Cancer Care Ontario, Toronto, ON; Dr. Arun Mathur (Surgeon), Oshawa Clinic, Oshawa, ON; Dr. Edward Matsumoto (Surgeon), St. Joseph's Hospital, Hamilton, ON; Linda Mayhew, Research Coordinator; Program in Evidence-based Care, McMaster University, Hamilton, ON; Dr. Tom McGowan (Radiation Oncology), Credit Valley Hospital, Mississauga, ON; Dr. Robin McLeod, Quality Lead, Cancer Care Ontario, Toronto, ON; Dr. Sheila McNair, Assistant Director, Program in Evidence-based Care, McMaster University, Hamilton, ON; Dr. Christopher Morash (Surgeon), The Ottawa Hospital - Civic Campus, Ottawa, ON; Dr. Madeleine Moussa (Pathologist), London Health Sciences Centre, London, ON; Dr. Linda Rabeneck, RVP, Toronto Sunnybrook Regional Cancer Centre, Toronto, ON; Bryan Rumble, Research Coordinator, Program in Evidence-based Care, McMaster University, Hamilton, ON; Dr. Thomas Short (Surgeon), Credit Valley Medical Arts Centre, Mississauga, ON; Dr. John Srigley (Pathologist), The Credit Valley Hospital, Mississauga, ON; Dr. John Tsihlias (Surgeon), William Osler Health Centre, Etobicoke, ON; Eric Winquist (Medical Oncology), London Health Sciences Centre, London, ON

Competing interests: None declared.

This paper has been peer-reviewed.

\section{References}

1. Neutel $\mathrm{Cl}, \mathrm{Gao}$ RN, Blood PA, et al. Trends in prostate cancer incidence, hospital utilization and surgical procedures, Canada, 1981-2000. Can J Public Health 2006;97:177-82

2. Neutel $\mathrm{Cl}$, Gao RN, Blood PA, et al. The changing age distribution of prostate cancer in Canada. Can I Public Health 2007;98:60-4.

3. Srigley JR. Key issues in handling and reporting radical prostatectomy specimens. Arch Pathol Lab Med 2006;130:303-17. 
Chin et al.

4. Srigley JR, Amin MB, Epstein Jl, et al. Prostate: protocol applies to invasive carcinomas of the prostate gland [monograph online]. Northfield, IL: College of American Pathologists; 2006. http://www.cap.org/apps/docs/committees/cancer/cancer_protocols/2006/prostate06_pw.pdf. Accessed December 1, 2009.

5. Gleave ME, Coupland D, Drachenberg D, et al. Ability of serum prostate-specific antigen levels to predict normal bone scans in patients with newly diagnosed prostate cancer. Urology 1996;47:708-12

6. Haukaus S, Roervik J, Halvorsen 0J, et al. When is bone scintigraphy necessary in the assessment of newly diagnosed, untreated prostate cancer? Br J Urol 1997;79:770-6.

7. D'Amico AV, Whittington R, Malkowicz SB, et al. Utilizing predictions of early prostate-specific antigen failure to optimize patient selection for adjuvant systemic therapy trials. J Clin Oncol 2000;18:3240-6.

8. Browman GP, Levine MN, Mohide EA, et al. The practice guidelines development cycle: a conceptual tool for practice guidelines development and implementation. J Clin Oncol 1995;13:502-12.

9. Chin JL, Srigley J, Mayhew LA, et al. Guideline for Optimization of Surgical and Pathological Quality Performance for Radical Prostatectomy in Prostate Cancer Management: Surgical and Pathological Guidelines. http://www.cancercare.on.ca/toolbox/qualityguidelines/din-program/surgery-ebs/. Accessed December 2, 2009

10. Rapp DE, Orvieto MA, Lucioni A, et al. Intra-operative prostate examination: predictive value and effect on margin status. BJU Int 2005;96:1005-8.

11. Salomon L, Anastasiadis AG, Johnson CW, et al. Seminal vesicle involvement after radical prostatectomy: predicting risk factors for progression. Urology 2003;62:304-9.

12. Guillonneau B, Cathelineau X, Doublet JD, et al. Laparoscopic radical prostatectomy: assessment after 550 procedures. Crit Rev Oncol Hematol 2002:43:123-33.

13. Guillonneau B, el-Fettouh $\mathrm{H}$, Baumert $\mathrm{H}$, et al. Laparoscopic radical prostatectomy: oncological evaluation after 1,000 cases a Montsouris Institute. J Urol 2003;169:1261-6.

14. Rabbani F, Bastar A, Fair WR. Site specific predictors of positive margins at radical prostatectomy: an argument for risk based modification of technique. [see comment]. J Urol 1998;160:1727-33.

15. Swindle $P$, Eastham JA, Ohori $M$, et al. Do margins matter? The prognostic significance of positive surgical margins in radical prostatectomy specimens. J Urol 2005;174:903-7.

16. Alsikafi NF, Brendler CB. Surgical modifications of radical retropubic prostatectomy to decrease incidence of positive surgical margins. J Urol 1998;159:1281-5.

17. Eastham JA, Kattan MW, Riedel E, et al. Variations among individual surgeons in the rate of positive surgical margins in radical prostatectomy specimens. J Urol 2003;170(6:Pt 1):2292-5.

18. Khan MA, Partin AW, Mangold LA, et al. Probability of biochemical recurrence by analysis of pathologic stage, Gleason score, and margin status for localized prostate cancer. Urology 2003;62:866-71.

19. Richman M, McLaughlin S, Maygarden S, et al. Initial incision of lateral pelvic fascia and early ligation of vascular pedicles during radical prostatectomy: potential to reduce positive margin rates. BJU Int 2005:95:40-5

20. Sofer M, Hamilton-Nelson KL, et al. Positive surgical margins after radical retropubic prostatectomy: the influence of site and number on progression. J Urol 2002;167:2453-6.

21. Vis AN, Schroder FH, van der Kwast TH. The actual value of the surgical margin status as a predictor of disease progression in men with early prostate cancer. Eur Urol 2006;50:258-65.

22. Cohn JH, El-Galley R. Radical prostatectomy in a community practice. J Urol 2002;167:224-8.

23. Graefen $M$, Hammerer $P$, Michl $U$, et al. Incidence of positive surgical margins after biopsy-selected nerve-sparing radical prostatectomy. Urology 1998;51:437-42.

24. Martinez-Pineiro L, Caceres F, Sanchez C, et al. Learning curve of laparoscopic radical prostatectomy in a university teaching hospital: experience after the first 600 cases. Eur Urol Suppl 2006;5:914-24.

25. Orvieto MA, Alsikafi NF, Shathav AL, et al. Impact of surgical margin status on long-term cancer control after radical prostatectomy. BJU Int 2006;98:1199-203.

26. Palisaar RJ, Noldus J, Graefen M, et al. Influence of nerve-sparing (NS) procedure during radical prostatectomy (RP) on margin status and biochemical failure. Eur Urol 2005:47:176-84.

27. Salomon L, Anastasiadis AG, Antiphon P, et al. Prognostic consequences of the location of positive surgical margins in organ-confined prostate cancer. Urol Int 2003;70:291-6.

28. Marcovich R, Woino KJ, Wei JT, et al. Bladder neck-sparing modification of radical prostatectomy adversely affects surgical margins in pathologic T3a prostate cancer. Urology 2000;55:904-8.

29. Blute ML, Bostwick DG, Bergstralh EJ, et al. Anatomic site-specific positive margins in organ-confined prostate cancer and its impact on outcome after radical prostatectomy. Urology 1997:50:733-9.

30. Deliveliotis C, Protogerou V, Alargof E, et al. Radical prostatectomy: bladder neck preservation and puboprostatic ligament sparing —effects on continence and positive margins. Urology 2002;60:855-8.

31. Kausik SJ, Blute ML, Sebo TJ, et al. Prognostic significance of positive surgical margins in patients with extraprostatic carcinoma after radical prostatectomy. Cancer 2002; $95: 1215-9$.

32. Sofer $\mathrm{M}$, Hamilton-Nelson $\mathrm{KL}$, et al. Risk of positive margins and biochemical recurrence in relation to nerve-sparing radical prostatectomy. J Clin Oncol 2002;20:1853-8.
33. Ward JF, Zincke $\mathrm{H}$, Bergstralh $\mathrm{EJ}$, et al. The impact of surgical approach (nerve bundle preservation versus wide local excision) on surgical margins and biochemical recurrence following radical prostatectomy. J Urol 2004;172(4 Pt 1):1328-32.

34. Aydin H, Tsuzuki T, Hernandez D, et al. Positive proximal (bladder neck) margin at radical prostatectomy confers greater risk of biochemical progression. Urology 2004;64:551-5.

35. Pettus JA, Weight CJ, Thompson CJ, et al. Biochemical failure in men following radical retropubic prostatectomy: impact of surgical margin status and location. J Urol 2004;172:129-32.

36. Guazzoni G, Cestari A, Naspro R, et al. Intra- and peri-operative outcomes comparing radical retropubic and laparoscopic radical prostatectomy: results from a prospective, randomised, single-surgeon study. Eur Urol 2006;50:98-104

37. Begg CB, Riedel ER, Bach PB, et al. Variations in morbidity after radical prostatectomy. N Engl J Med 2002;346:1138-44

38. Kundu SD, Roehl KA, Eggener SE, et al. Potency, continence and complications in 3,477 consecutive radical retropubic prostatectomies. J Urol 2004;172(6 pt 1):2227-31.

39. Bianco $\mathrm{FJ}$, Jr., Riedel ER, Begg CB, et al. Variations among high volume surgeons in the rate of complications after radical prostatectomy: further evidence that technique matters. [see comment]. I Urol 2005;173:2099-103.

40. Ponholzer A, Brossner C, Struhal G, et al. Lower urinary tract symptoms, urinary incontinence, sexual function and quality of life after radical prostatectomy and external beam radiation therapy: real life experience in Austria. World I Urol 2006;24:325-30.

41. Catalona WJ, Carvalhal GF, Mager DE, et al. Potency, continence and complication rates in 1,870 consecutive radical retropubic prostatectomies. J Urol 1999;162:433-8.

42. Burkhard FC, Kessler TM, Fleischmann A, et al. Nerve sparing open radical retropubic prostatectomydoes it have an impact on urinary continence? J Urol 2006;176:189-95.

43. Lowe BA. Comparison of bladder neck preservation to bladder neck resection in maintaining postrostatectomy urinary continence. Urology 1996;48:889-93

44. Anastasiadis AG, Benson MC, Rosenwasser MP, et al. Cavernous nerve graft reconstruction during radical prostatectomy or radical cystectomy: safe and technically feasible. Prostate Cancer Prostatic Dis 2003;6:56-60

45. Heidenreich A, Varga Z, Von KR. Extended pelvic lymphadenectomy in patients undergoing radical prostatectomy: high incidence of lymph node metastasis. [see comment]. J Urol 2002;167:1681-6.

46. Lance RS, Freidrichs PA, Kane C, et al. A comparison of radical retropubic with perineal prostatectomy for localized prostate cancer within the Uniformed Services Urology Research Group. BJU Int 2001:87:61-5.

47. Maffezzini $M$, Seveso $M$, Taverna $G$, et al. Evaluation of complications and results in a contemporary series of 300 consecutive radical retropubic prostatectomies with the anatomic approach at a single institution. Urology 2003;61:982-6.

48. Tewari A, Peabody JO, Fischer $M$, et al. An operative and anatomic study to help in nerve sparing during laparoscopic and robotic radical prostatectomy. Eur Urol 2003;43:444-54

49. Lee DK, Allareddy V, O'Donnell MA, et al. Does the interval between prostate biopsy and radical prostatectomy affect the immediate postoperative outcome? BJU Int 2006;97:48-50.

50. Nuttall GA, Cragun MD, Hill DL, et al. Radical retropubic prostatectomy and blood transfusion. Mayo Clin Proc 2002;77:1301-5.

51. Cannon GM Jr, Pound CR, Landsittel DP, et al. Perineural invasion in prostate cancer biopsies is not associated with higher rates of positive surgical margins. Prostate 2005;63:336-40.

52. Lilleby W, Fossa SD, Waehre HR, et al. Long-erm morbidity and quality of life in patients with localized prostate cancer undergoing definitive radiotherapy or radical prostatectomy. Int J Radiat Oncol Biol Phys 1999:43:735-43

53. Bianco FJ, Jr., Scardino PT, Eastham JA. Radical prostatectomy: long-term cancer control and recovery of sexual and urinary function ("trifecta"). Urology 2005;66 (5 Suppl):83-94.

54. Clark T, Parekh DJ, Cookson MS, et al. Randomized prospective evaluation of extended versus limited lymph node dissection in patients with clinically localized prostate cancer. J Urol 2003;169:145-7.

55. Briganti A, Chun FKH, Salonia A, et al. Complications and other surgical outcomes associated with extended pelvic lymphadenectomy in men with localized prostate cancer. Eur Urol 2006;50:1006-13.

56. Berglund RK, Sadetsky N, Duchane J, et al. Limited pelvic lymph node dissection at the time of radical prostatectomy does not affect the 5-year failure rates for low, intermediate and high risk prostate cancer: results from CaPSURE. J Urol 2007:177:526-30.

57. Joslyn SA, Konety BR. Impact of extent of lymphadenectomy on survival after radical prostatectomy for prostate cancer. Urology 2006;68:121-5.

58. Masterson TA, Bianco FJ Jr, Vickers AJ, et al. The association between total and positive lymph node counts, and disease progression in clinically localized prostate cancer. J Urol 2006; 175:1320-4.

59. Allaf ME, Palapattu GS, Trock BJ, et al. Anatomical extent of lymph node dissection: impact on men with clinically localized prostate cancer. J Urol 2004;172(5 Pt 1):1840-4. 
60. Bhatta-Dhar N, Reuther AM, Zippe C, et al. No difference in six-year biochemical failure rates with or without pelvic lymph node dissection during radical prostatectomy in low-risk patients with localized prostate cancer. Urology 2004;63:528-31.

61. Fergany A, Kupelian PA, Levin HS, et al. No difference in biochemical failure rates with or without pelvic lymph node dissection during radical prostatectomy in low-risk patients. Urology 2000;56:92-5.

62. DiMarco DS, Zincke H, Sebo TJ, et al. The extent of lymphadenectomy for pTXNO prostate cancer does not affect prostate cancer outcome in the prostate specific antigen era. J Urol 2005; 173:1121-5.

63. Bader $P$, Burkhard $F C$, Markwalder $R$, et al. Is a limited lymph node dissection an adequate staging procedure for prostate cancer? J Urol 2002;168:514-8.

64. Bader P, Burkhard $\mathrm{FC}$, Markwalder R, et al. Disease progression and survival of patients with positive lymph nodes after radical prostatectomy. Is there a chance of cure? [see comment]. J Urol. 2003 Mar, 169(3):849-54.

65. Parra RO, Isorna S, Perez MG, et al. Radical perineal prostatectomy without pelvic lymphadenectomy: selection criteria and early results. J Urol 1996;155:612-5.

66. Burkhard FC, Bader P, Schneider E, et al. Reliability of preoperative values to determine the need for lymphadenectomy in patients with prostate cancer and meticulous lymph node dissection. Eur Urol 2002; $42: 84-92$

67. Pagliarulo V, Hawes D, Brands FH, et al. Detection of occult lymph node metastases in locally advanced node-negative prostate cancer. J Clin Oncol 2006;24:2735-42.
68. Rogers E, Gurpinar T, Dillioglugil 0 , et al. The role of digital rectal examination, biopsy Gleanson sum and prostate-specific antigen in selecting patients who require pelvic lymph node dissections for prostate cancer. Br J Urol 1996:78:419-25.

69. Alagiri $M$, Colton MD, Seidmon EJ, et al. The staging pelvic lymphadenectomy: implications as an adjunctive procedure for clinically localized prostate cancer. Br I Urol 1997;80:243-6.

70. Miyake H, Sakai I, Harada K, et al. Is a limited lymphadenectomy targeting obturator nodes alone an adequate procedure for Japanese men undergoing radical prostatectomy? Int I Urol 2005; 12:739-44.

71. Wawroschek F, Wagner T, Hamm M, et al. The influence of serial sections, immunohistochemistry, and extension of pelvic lymph node dissection on the lymph node status in clinically localized prostate cancer. Eur Urol 2003;43:132-6.

72. Weckermann D, Wawroschek F, Harzmann R. Is there a need for pelvic lymph node dissection in low risk prostate cancer patients prior to definitive local therapy? Eur Urol 2005;47:45-51.

73. Weckermann D, Goppelt $M$, Dorn $R$, et al. Incidence of positive pelvic lymph nodes in patients with prostate cancer, a prostate-specific antigen (PSA) level of $<10 \mathrm{ng} / \mathrm{mL}$ and biopsy Gleason score of $<6$, and their influence on PSA progressionffree survival after radical prostatectomy. BJU Int 2006;97:1173-8.

Correspondence: Dr. Joseph Chin, Regional Head of Surgical Oncology, London Health Sciences Centre, 800 Commissioners Rd. East, London, 0N N6A 4G5; Joseph.chin@|hsc.on.ca

\section{CUA MEMBERS!}

\section{Seek accreditation through our office}

The CUA Office of Education (OE) encourages you to seek accreditation through our office which, along with a simplified and quick process, includes:
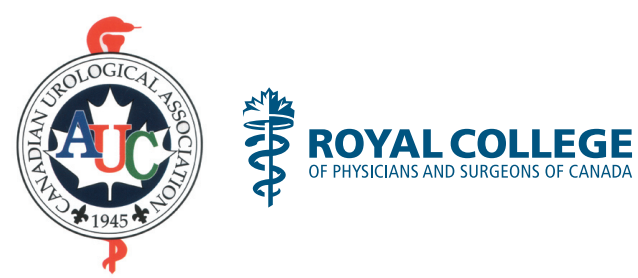

- access to members via e-mail blasts

- posting of events in CUAJ and on the CUA and CUAJ websites

- a direct link to the Royal College MAINPORT site

For a complete listing of fees, please visit www.cua.org

Please contact:

Nadia P. Pace

Project Manager

CUA Office of Education

nadia.pace@cua.org 